\title{
Report on evaluation, verification and assessment of porosity migration model in fast reactor MOX fuel
}

\author{
S. R. Novascone and J. W. Peterson \\ September 2016
}

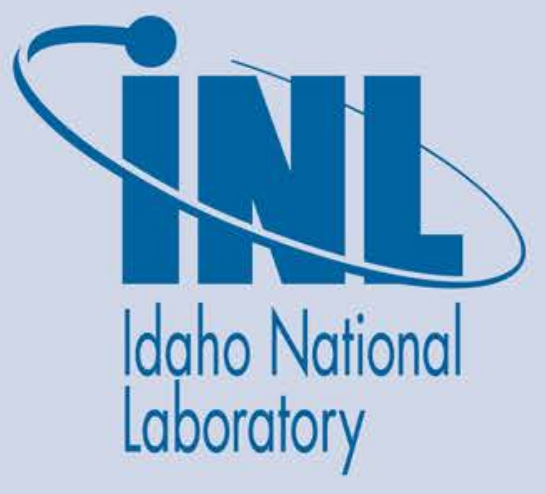

The INL is a U.S. Department of Energy National Laboratory operated by Battelle Energy Alliance 
INL/EXT-16-39927

\section{Report on evaluation, verification and assessment of porosity migration model in fast reactor MOX fuel}

S. R. Novascone and J. W. Peterson

September 2016

Idaho National Laboratory
Idaho Falls, Idaho 83415

http://www.inl.gov

Prepared for the

U.S. Department of Energy

Assistant Secretary for _, OR Office of

Under DOE Idaho Operations Office

Contract DE-AC07-05ID14517 


\section{INL REPORT}

INL

Unlimited Release

Printed September 2016

\section{Report on evaluation, verification and assessment of porosity migration model in fast reactor MOX fuel}

S. R. Novascone

J. W. Peterson

Revised September 13, 2016

Prepared by

Idaho National Laboratory

Idaho Falls, Idaho 83415

The Idaho National Laboratory is a multiprogram laboratory operated by

Battelle Energy Alliance for the United States Department of Energy

under DOE Idaho Operations Office. Contract DE-AC07-05ID14517.

Approved for public release; further dissemination unlimited.

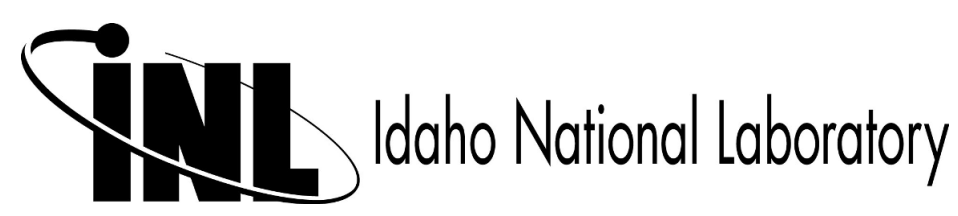


Issued by the Idaho National Laboratory, operated for the United States Department of Energy by Battelle Energy Alliance.

NOTICE: This report was prepared as an account of work sponsored by an agency of the United States Government. Neither the United States Government, nor any agency thereof, nor any of their employees, nor any of their contractors, subcontractors, or their employees, make any warranty, express or implied, or assume any legal liability or responsibility for the accuracy, completeness, or usefulness of any information, apparatus, product, or process disclosed, or represent that its use would not infringe privately owned rights. Reference herein to any specific commercial product, process, or service by trade name, trademark, manufacturer, or otherwise, does not necessarily constitute or imply its endorsement, recommendation, or favoring by the United States Government, any agency thereof, or any of their contractors or subcontractors. The views and opinions expressed herein do not necessarily state or reflect those of the United States Government, any agency thereof, or any of their contractors.

Printed in the United States of America. This report has been reproduced directly from the best available copy.

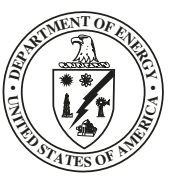


INL

Unlimited Release

Printed September 2016

\title{
Report on evaluation, verification and assessment of porosity migration model in fast reactor MOX fuel
}

\author{
S. R. Novascone \\ Department of Fuel Modeling and Simulation (C650) \\ Nuclear Science \& Technology Directorate \\ Idaho National Laboratory \\ P.O. Box 1625 \\ Idaho Falls, ID 83415-3840 \\ J. W. Peterson \\ Department of Modeling and Simulation (C090) \\ Nuclear Science \& Technology Directorate \\ Idaho National Laboratory \\ P.O. Box 1625 \\ Idaho Falls, ID 83415-3840 \\ Revised September 13, 2016
}




\begin{abstract}
This report documents the progress of simulating pore migration in ceramic $\left(\mathrm{UO}_{2}\right.$ and mixed oxide or MOX) fuel using BISON. The porosity field is treated as a function of space and time whose evolution is governed by a custom convection-diffusion-reaction equation (described here) which is coupled to the heat transfer equation via the temperature field. The porosity is initialized to a constant value at every point in the domain, and as the temperature (and its gradient) are increased by application of a heat source, the pores move up the thermal gradient and accumulate at the center of the fuel in a time-frame that is consistent with observations from experiments. There is an inverse dependence of the fuel's thermal conductivity on porosity (increasing porosity decreases thermal conductivity, and vice-versa) which is also accounted for, allowing the porosity equation to couple back into the heat transfer equation. Results from an example simulation are shown to demonstrate the new capability.
\end{abstract}




\section{Acknowledgments}

Special thanks to Steve Hayes, Rich Williamson, Jason Hales, and Pavel Medvedev for their input. Also, thanks go to Russell Gardner for help with the plots. 


\section{Contents}

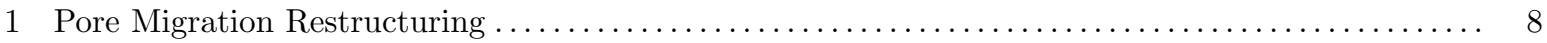

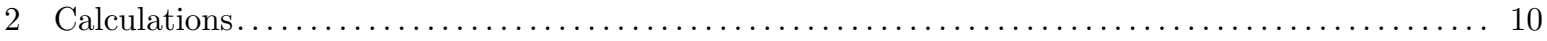

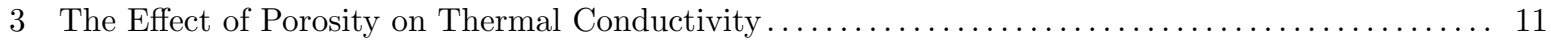

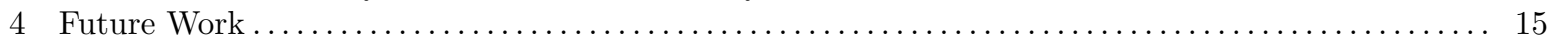




\section{Figures}

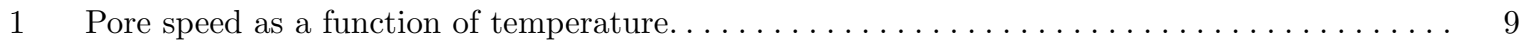

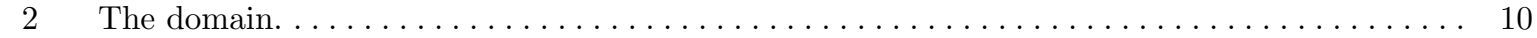

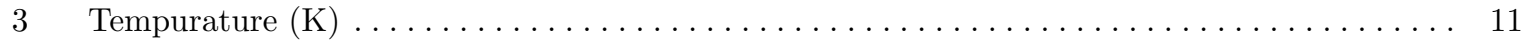

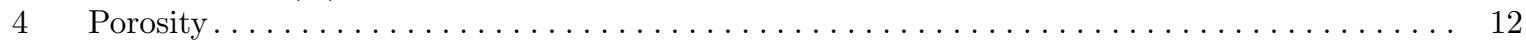

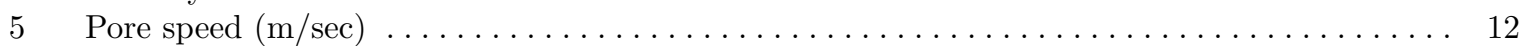

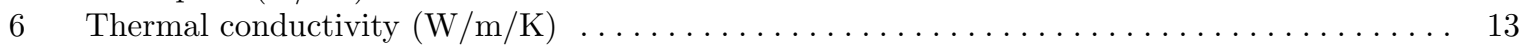

7 Preliminary porosity contour plot of the B-14 rod ptm001 experiment (image is not to scale) . 14 


\section{Pore Migration Restructuring}

Much of the discussion here is based on various literature sources [1-6] and email correspondence with Takayuki Ozawa from JAEA. After start-up and attainment of full-power and nominal operating fuel temperature, ceramic fuel $\left(\mathrm{UO}_{2}\right.$ and $\left.\mathrm{MOX}\right)$ experiences meso-scale restructuring, which has a profound effect on the bulk properties of the fuel. Here, restructuring means that the fuel pellet develops distinct regions. Going from the center of the fuel outward in sequence, these regions are characterized by a central hole, a region of increased density, then a region with aligned grains and un-restructured grains. These regions form radial iso-surfaces due to restructuring of the fuel, which depends on temperature and its gradient.

The salient feature of all this restructuring, for this work, is the movement of pores. Pores introduced during fabrication are generally greater than $1 \mu \mathrm{m}$ in size, and are uniformly distributed throughout the pellet. Pores move toward the center of the pellet where they eventually coalesce into a large central void. Speculation regarding the manner in which the central void forms has lead to the concept of a vapor transport mechanism in which fuel at the surface of a pore closest to the fuel center vaporizes, travels across the pore, and finally condenses on the surface of the pore furthest from the fuel center. The result is a net flux of pores toward the pellet center.

To begin accounting for pore migration, a preliminary model in BISON tracks porosity as a field variable using a PDE that describes continuity of pores. This model can be used to predict the central hole formation and inform thermal conductivity models that are based on porosity. Restructuring also depends upon the deviation of oxygen from stoichiometric amount, average size of grains, plutonium content, and actinide concentration.

The PDE that describes pore migration in this work is,

$$
\frac{\partial p}{\partial t}+\nabla \cdot\left(v_{p} p \nabla T-\nu \nabla p\right)=0
$$

In Eqn. (1), $p$ is the porosity, $v_{p}$ is the pore velocity, $T$ is the temperature, and $\nu$ is the diffusion coefficient. Usually, the temperature gradient is included in the pore velocity term, but here, the temperature gradient is written separately to emphasize the dependence of pore migration on temperature gradient. Eqn. (1) is akin to mass conservation and diffusion, except applied to pores. Rather than thinking of pores as voids (at least for now), think of porosity as a quantity that is transported within the domain according to well-established principles of conservation. Since pores apparently move through fuel by way of a vapor transport mechanism, applying a conservation law seems intuitive. Pores may or may not diffuse through the fuel, however diffusion is included in the present model for numerical stability reasons, and to ensure that reasonable steady-state solutions can be obtained for the application under consideration. The value of the diffusion coefficient and its dependence on other field properties, such as temperature, is an area that requires more investigation.

Pore velocity, $v_{p}$, is a highly uncertain quantity, however some order of magnitude approximations are possible. A literature review uncovered several equations for pore velocity. A new equation, in a yet-to-be published article from Takayuki Ozawa shows pore velocity to be a function of the sum of partial pressures and corresponding derivatives with respect to temperature of the mixed oxide constituents $\left(\mathrm{UO}_{2}, \mathrm{PuO} 2\right.$, $\mathrm{UO}, \mathrm{U}, \mathrm{UO}_{3}, \mathrm{PuO}, \mathrm{Pu}, \mathrm{AmO}_{2}, \mathrm{Am}, \mathrm{AmO}$, and $\mathrm{O}_{2}$ ). The authors initially attempted to implement Ozawa's expression, however, difficulties with the computation of the partial pressure of oxygen, which requires a separate nonlinear solution, lead to the selection of a different expression for pore velocity from Nichols [5], given by:

$$
v_{p}=\frac{K p_{0} \exp (-H / R T)}{P T^{3 / 2}} \nabla T
$$

In Eqn. (2), $K$ and $p_{0}$ are material constants, $H$ is the heat of vaporization, $R$ is the universal gas constant, $T$ is temperature, and $P$ is pressure in the pore. The sub-expression $p_{0} \exp (-H / R T)$ is the sum of partial pressures of the uranium and plutonium vapor species. In this work, only $\mathrm{UO}_{2}$ is considered. Lackey [6] has an expression that accounts for plutonium constituents, which will be implemented next FY. 
A plot of pore speed vs. temperature is shown in the following figure with an assumed temperature gradient of constant $1 \mathrm{e} 5 \mathrm{~K} / \mathrm{m}$.

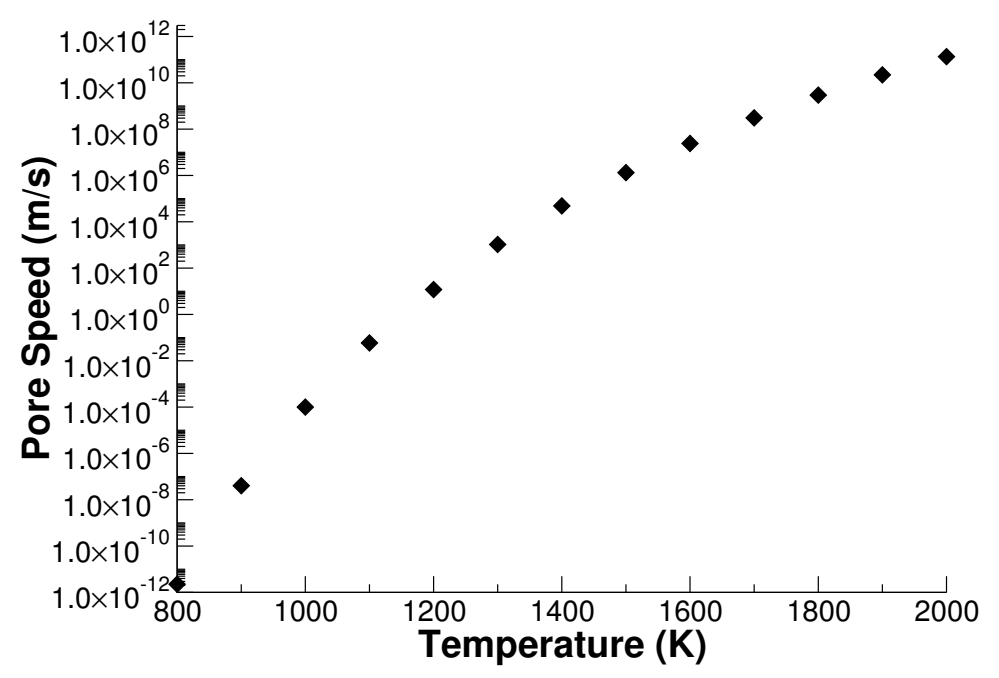

Figure 1: Pore speed as a function of temperature.

Note that Fig. 1 shows pore speeds that are unrealistically high even at expected fuel temperatures, which for fast fuel is about $1000-2000 \mathrm{~K}$. Thus, the model described by Eqn. (2) should only be considered "valid" up to an arbitrarily-chosen maximum velocity. In this work, an upper speed limit of $100 \mathrm{~m} / \mathrm{s}$ (regardless of temperature gradient strength, pore velocity is bounded by this limit) seems to give reasonable pore distribution. This is obviously an area that requires further investigation. Hopefully, our continued collaboration with the JAEA and NNL (UK) will provide guidance on this topic. 


\section{Calculations}

Results for a pore migration calculation using Eqns. (1) and (2) are shown in this section. For this demonstration problem, a quarter-symmetry 2D Cartesian cylindrical domain was used. See Fig. 2 for details of the mesh quality and connectivity, the radius of the cylinder is taken to be $2 \mathrm{~mm}$.

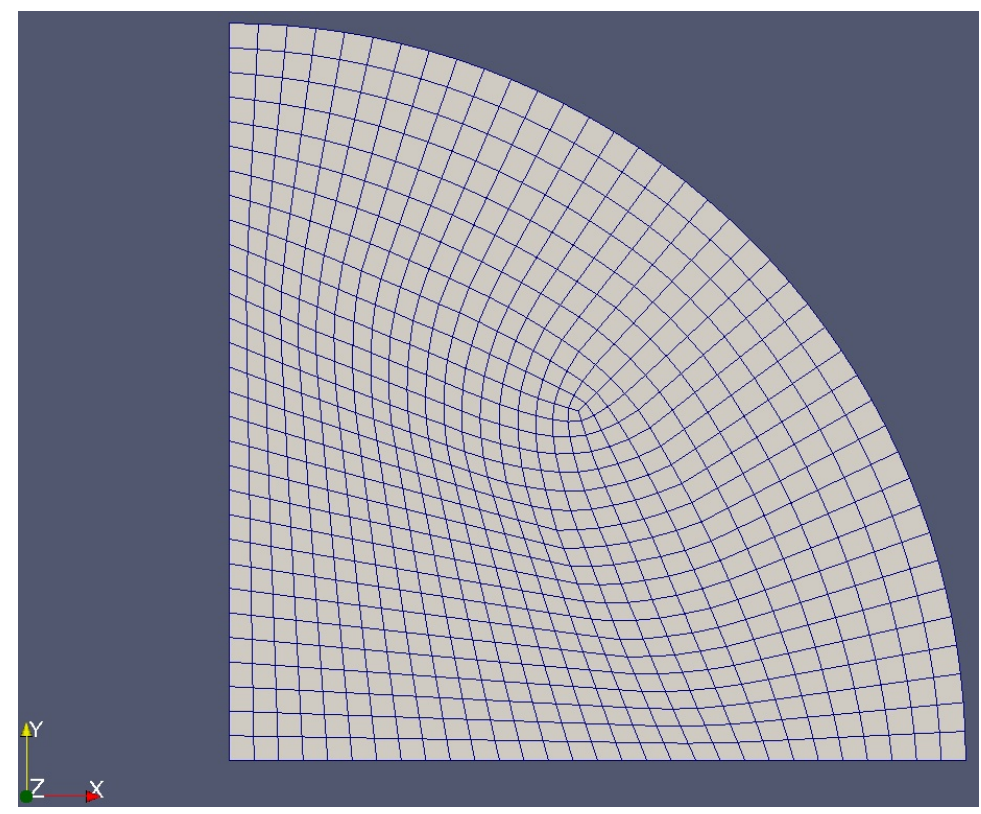

Figure 2: The domain.

The equations solved simultaneously are:

$$
\begin{array}{r}
\rho C \frac{\partial T}{\partial t}-\nabla \cdot k \nabla T-q=0 \\
\frac{\partial p}{\partial t}+\nabla \cdot\left(v_{p} p \nabla T-\nu \nabla p\right)=0
\end{array}
$$

where $\rho$ is density, $C$ is heat capacity, $k$ is thermal conductivity (from Ozawa and is a function of porosity and temperature), $T$ is temperature, $q$ is the heat source term. The terms in Eqn. (4) are as described previously.

The boundary conditions are $T=1000 \mathrm{~K}$ (Dirichlet, fixed) and $\frac{\partial p}{\partial n}=0$ (no flux) on the outside of the cylinder, and symmetry along the internal radial lines. The source term is defined by a linear heat rate that ramps from zero to $40 \mathrm{~kW} / \mathrm{m}$ in 10,000 seconds, then stays constant for the remainder of the simulation. The initial condition for the porosity field is $p=1$ everywhere, and the diffusion coefficient $(\nu$ in Eqn. (4)) is taken to be 1 as well.

The porosity variable can reasonably be thought of as a dimensionless quantity, with $p=0$ representing solid material with no pores, and $p=1$ representing empty space (void). In this convention, values of $p$ larger than 1 and smaller than 0 obviously don't make sense. Unfortunately, with the chosen boundary conditions and physical model described here (a Neumann problem is prescribed for the porosity equation), there is nothing which a priori limits the porosity to values in the range $[0,1]$. Instead, we can imagine a "normalized" porosity value which is used by material properties such as thermal conductivity, and which always lies in the range $[0,1]$ regardless of what the computed porosity may be. In this context, the actual value of the porosity computed by Eqn. (4) is of less importance than the overall dynamic range of the variable. 


\section{The Effect of Porosity on Thermal Conductivity}

A thermal conductivity model for MOX fuel was used in this simulation from Kato et al. [7]. In the calculations shown here, the magnitude of thermal conductivity was decreased as the porosity increased in a fashion similar to that described in the following equation, which is consistent with other modeling efforts.

$$
f_{p}=\frac{1-p}{1+0.5 p}
$$

In Eqn. (5), when the porosity is $0, f_{p}$ is 1 . As the porosity increases to $1, f_{p}$ approaches 0 . To account for the effect of porosity on thermal conductivity, $f_{p}$ is multiplied by the thermal conductivity. In the current approach, Eqn. (5) isn't used, but the same effect is captured. The porosity starts at 1, and increases or decreases according to Eqn. (4). As the porosity increases by an order of magnitude, $f_{p}$ decreases by an order of magnitude in a linear fashion similar to Eqn. (5). In other words, when $p$ is $1, f p$ is 1 and when $p$ is $10, f p$ is 0.1 .

Figs. 3-6 show contour plots for temperature, porosity, pore velocity, and thermal conductivity, respectively at the end of the simulation.

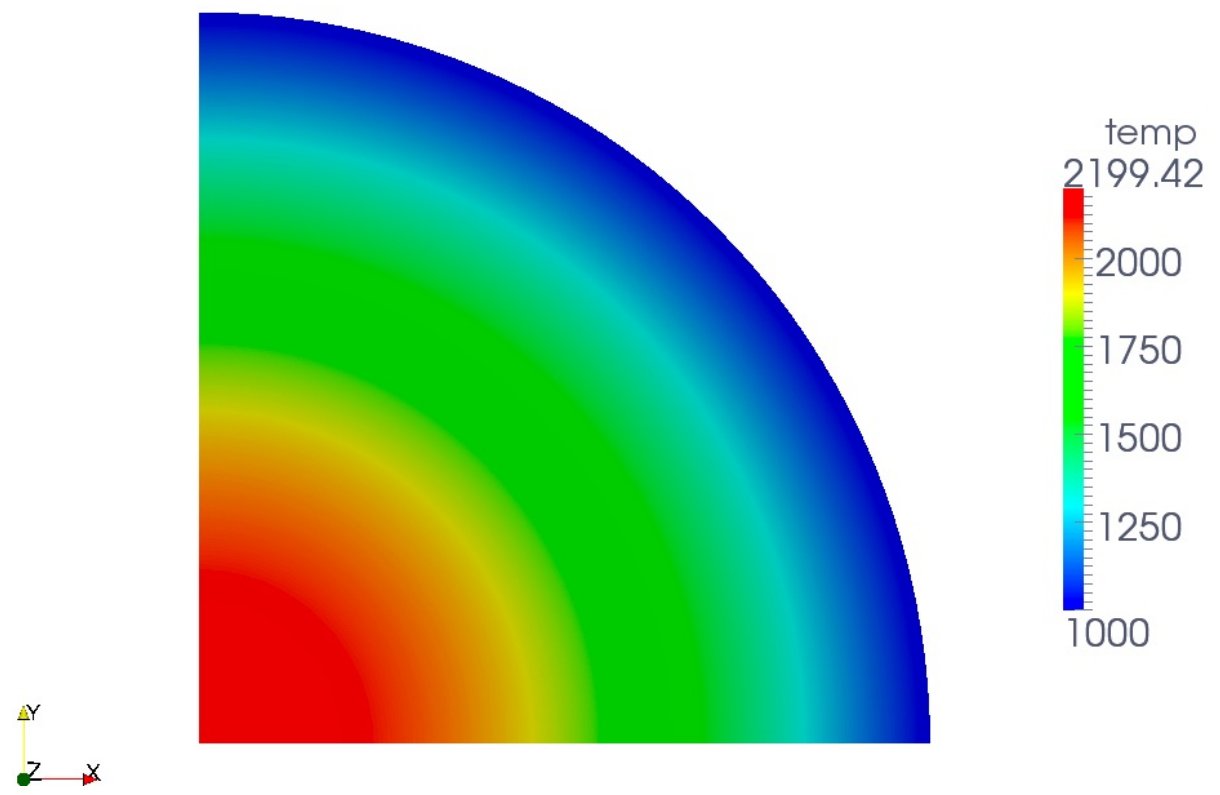

Figure 3: Tempurature (K)

The simulation runs well with small time steps during the power ramp, and after the power ramp large time steps $\left(\approx 10^{6}\right.$ seconds) can be taken. This approach was used in simulating the B-14 experiment with rod ptm001. Preliminary results of the simulation are shown in Fig. 7. Note the RZ symmetry. The stepped or jagged look of the plot is due to a prescribed axial profile of the same shape. 


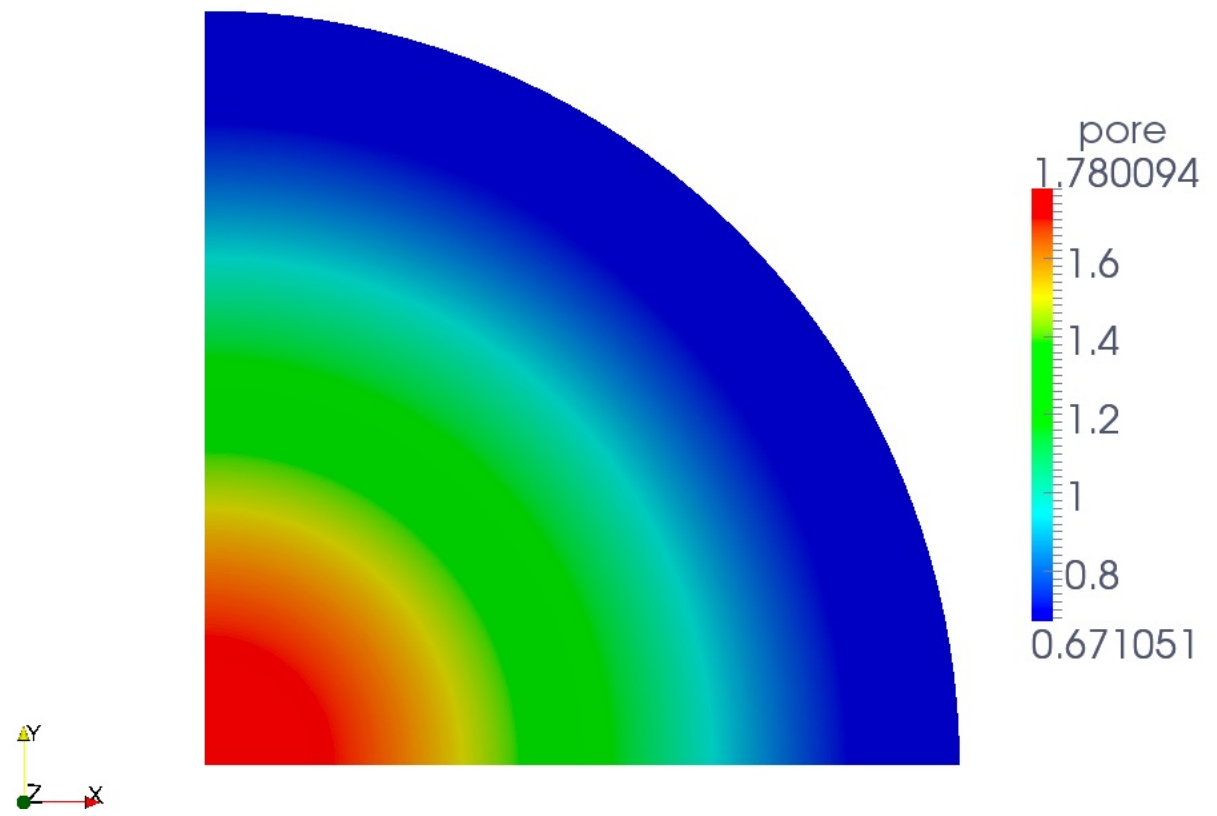

Figure 4: Porosity

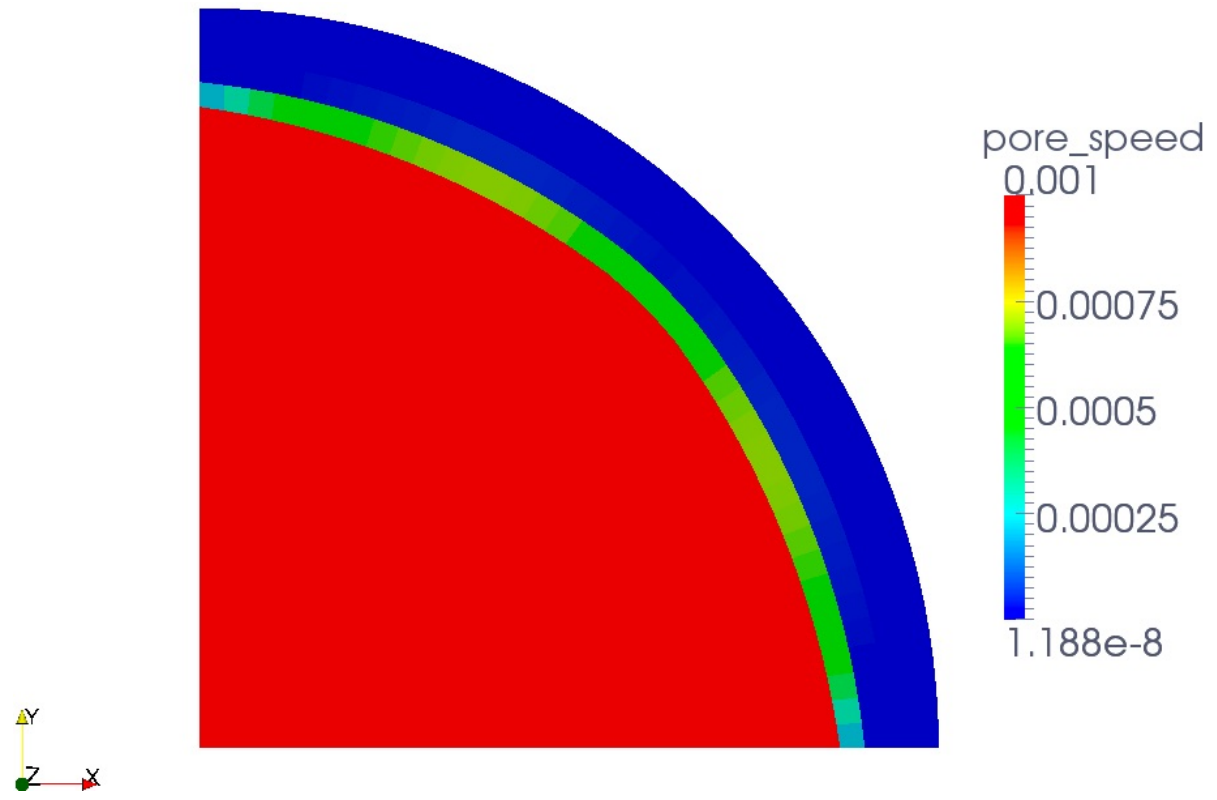

Figure 5: Pore speed $(\mathrm{m} / \mathrm{sec})$ 


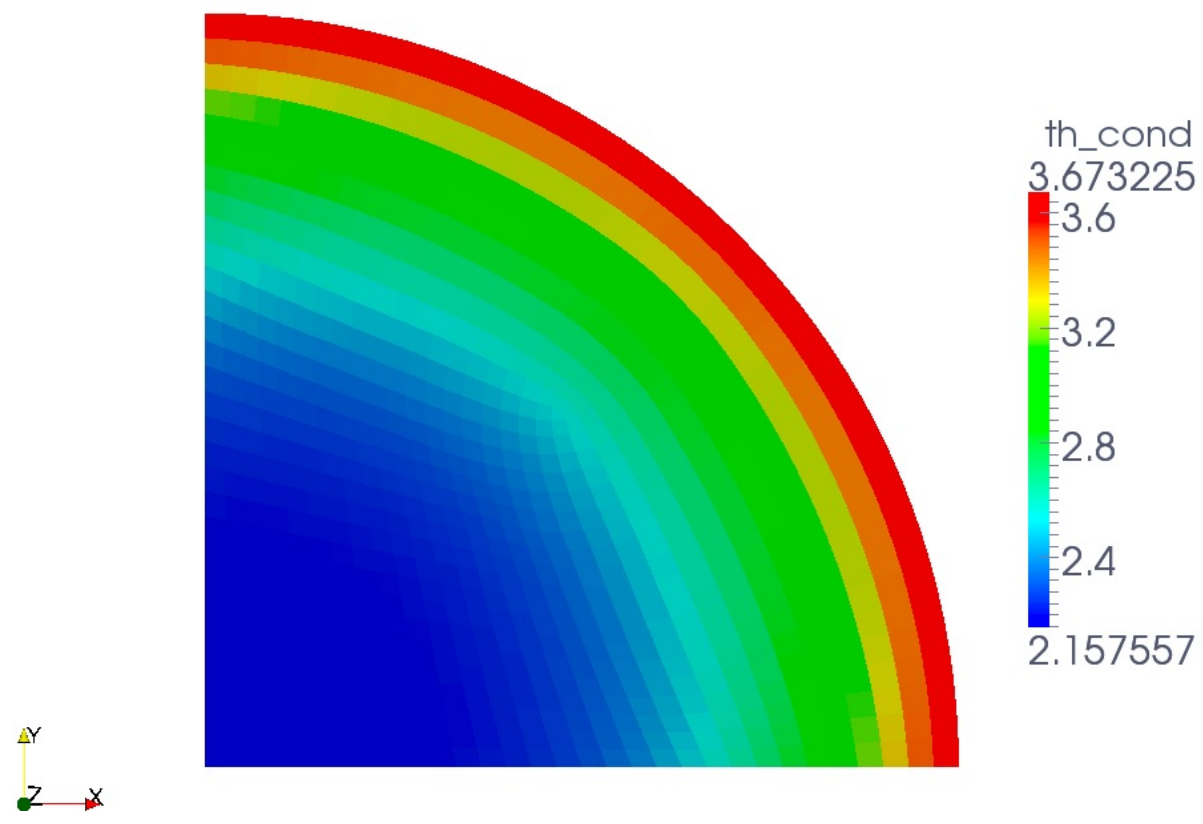

Figure 6: Thermal conductivity $(\mathrm{W} / \mathrm{m} / \mathrm{K})$ 

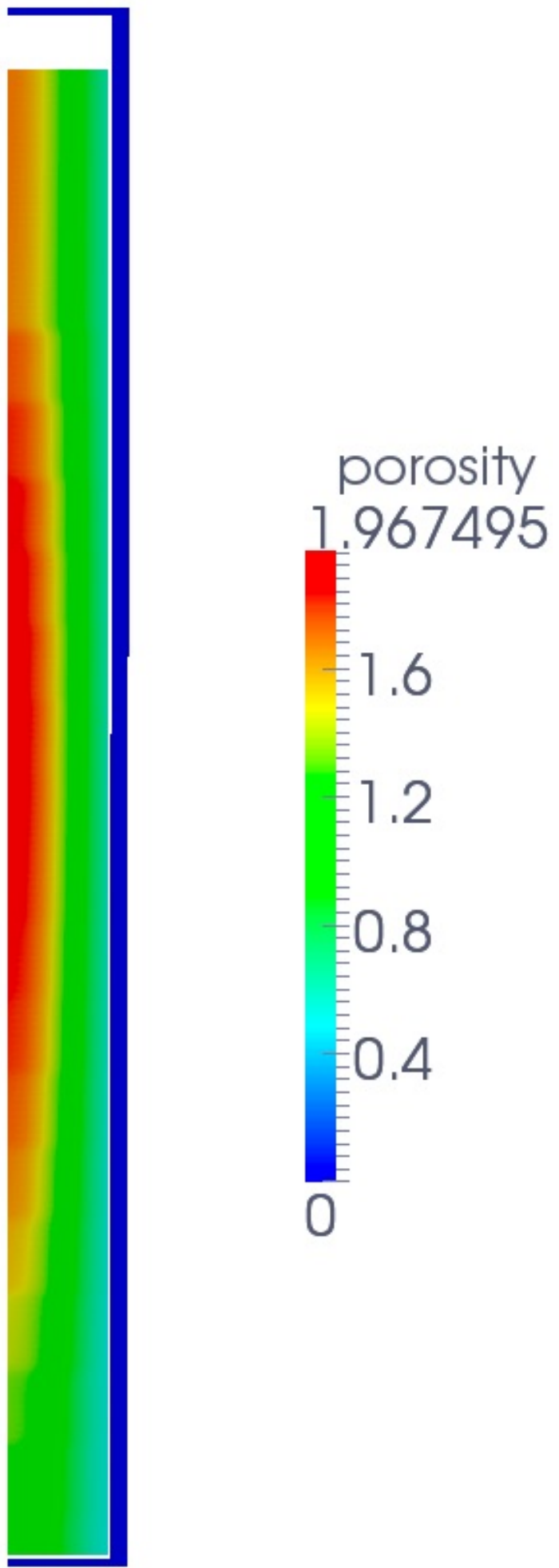

Figure 7: Preliminary porosity contour plot of the B-14 rod ptm001 experiment (image is not to scale) 


\section{Future Work}

Several open issues remain for this model, and they must be addressed for it to be of practical use to analysts. The feasibility of the "porosity normalization" approach must be better-understood, as well as the consequences of employing a model in which porosity values larger than 1 are not precluded by the physics. It may also be possible to employ other configurations of boundary conditions that will somewhat ameliorate this issue.

The uncertainty surrounding the pore speed/temperature correlation, including the cutoff value beyond which Eqn. (2) is no longer valid, must be better quantified. Conflicting experimental observations, including evidence that pores only begin moving at temperatures above approximately $1300^{\circ} \mathrm{C}(1573 \mathrm{~K})[1]$, a temperature for which the pore correlation of Fig. 1 predicts an unrealistically high value, must also be reconciled. Finally, the pore speed correlation should also eventually include the effects of the mixed constituents, which requires a more sophisticated, nonlinear material model.

The choice to model porosity evolution with a convection-diffusion equation implies that the movement of pores is, in some sense, "reversible.". In other words, if the temperature (and corresponding gradient) were to return to its pre-power-ramp configuration, the porosity field would also return to its pre-power-ramp configuration, a situation which is not physically-realistic, as the central void formation process is known to be irreversible.

A short term solution to this issue is to only allow the porosity to increase in the material models, regardless of what the porosity field calculation predicts at any given time. It's not clear how this would affect the nature of the coupling between the equations, however, and doing so might introduce additional numerical difficulties. A longer term solution may be to incorporate the use of XFEM or element deletion. This latter approach (element deletion) has been used successfully in other contexts, but not yet for convection-diffusion models, and it is not known how easily it will couple with the other equations of fuels modeling. For example, once elements with a high enough porosity are deleted from the mesh, other quantities which are important for verification, such as conservation of porosity and energy, are no longer maintained in the usual sense. For these reasons, we are also investigating the possibility of employing different PDE models, especially those which inherently incorporate the concepts of hysteresis and irreversibility, in place of the convection-diffusion model. 


\section{References}

[1] Aydin Karahan. Modeling of thermo-mechanical and irradiation behavior of metallic and oxide fuels for sodium fast reactors. PhD thesis, Massachusetts Institute of Technology, June 2009.

[2] D. R. Olander. Fundamental aspects of nuclear reactor fuel elements. Technical Information Center, Energy Research and Development Administration, 1976.

[3] T. Ozawa and T. Abe. Development and verifications of fast reactor fuel design code CEPTAR. Nuclear Technology, 156:39-55, 2006.

[4] D. R. De Halas and G. R. Horn. Evolution of uranium dioxide structure during irradiation of fuel rods. J. Nucl. Mater., 8(2):207-220, 1963.

[5] F. A. Nichols. Theory of columnar grain growth and central void formation in oxide fuel rods. J. Nucl. Mater., 22:214-222, 1967.

[6] W. J. Lackey, F. J. Homan, and A. R. Olsen. Porosity and actinide redistribution during irradiation of (U,Pu) $\mathrm{O}_{2}$. Nuclear Technology, 16:120-142, October 1972.

[7] M. Kato, K. Maeda, T. Ozawa, M. Kashimura, and Y. Kihara. Physical properties and irradiation behavior analysis of np- and am-bearing mox fuels. Nuclear Science and Technology, 48(4):646-653, 2011. 


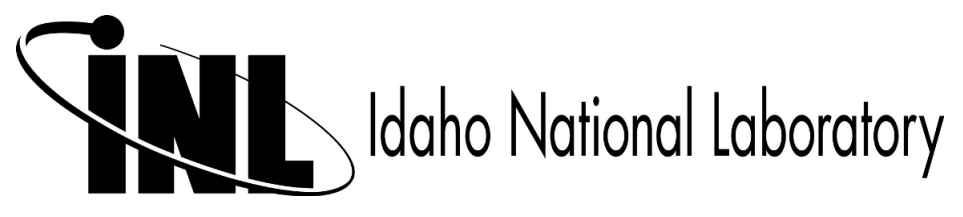

